\title{
COVIDetect (A Deep Neural Network for Detecting COVID-19 Cases from Chest CT-scan Images)
}

\author{
Md. Mobarak Hossain \\ PhD Fellow, Department of CSE, Dhaka University of Engineering and Technology, \\ Bangladesh \\ Muhammad Usama Islam \\ Lecturer, Department of CSE, Asian University of Bangladesh

\section{Dr. Mohammod AbulKashem} \\ Professor, Department of CSE, Dhaka University of Engineering and Technology, \\ Bangladesh \\ Corresponding Author: Muhammad Usama Islam \\ Corresponding Author Email: usamaislam@iut-dhaka.edu
}

\begin{abstract}
Coronavirus (COVID) has claimed numerous lives since its outbreak in late 2019. It is estimated that around 72 million people are affected by the virus and a toll on human life has reached 1.6 millions as of December 2020 making it one of the worst pandemic in recorded history. Having understood the importance of the pandemic, we devised a deep neural network model to classify COVID patients from non-COVID patients based on computerized tomography (CT) scan images with an accuracy of $76.5 \%$ that concluded our contribution to the growing pandemic.
\end{abstract}

Keywords: COVID-19, Coronavirus, Image Processing, Deep learning, Neural Network 


\section{Introduction}

\subsection{Rationale of the study}

The Coronavirus ( COVID-19) has impacted the lives of billions of people worldwide. Everyday due to its nature being of a pandemic, it has affected to a greater extent thus calling for aid from all over the scientific community. The research and development has expanded from care-coordination of COVID patients to care management to prevention guidelines. One of the interesting research works in this field is the early detection of COVID. The rationale behind early detection is, as early the COVID is being detected in the body, the sooner the care management supply chain begins to work thus minimizing the death toll of human life. The importance of the study goes beyond vaccination as current state of the art has a $95 \%$ prevention rate on vaccination which will take almost 1-2 years to vaccinate the entire world which makes this study an pertinent contribution to COVID detection, identification, classification and segmentation research field.

\subsection{Literature Review}

The COVID classification problem includes the identification of COVID presence in the body with the aid of sensor data, X-ray images, CT-images, Radiography images and so forth [1-4].Various research works has been carried out for the detection and classification of COVID-19[4,5].It is one of the challenging issue to understand the presence of COVID within an automated image. The most challenging part of this disease detection from image is to identify the difference between pneumonia image and COVIDimage which makes the task significantly hard.

The use of neural networks and big data has been explored in [6-10]. Ulhaq et.al.[6] conferred a survey literature that accumulates the effort of various computer vision approaches that has been applied to detect COVID. Zobaed and his colleagues[8] has explored how the big data can be stored and manipulated on the cloud of which the continuation of manipulation over wearable sensor data is performed by Quer and his team[9] where they have explored the wearable sensor data as an aiding method in detecting COVID.

The general diagnostic tests and its effects as well as the time consumption of .2 general diagnostic tests as opposed to antibody tests has been explored in [10]. An interesting analogy by Vandenberg et.al [10] and Bastos'steam[11] depicts 
that albeit antibody tests provided rapid results in testing but it suffers with low rate of specificity which is an important metric in identifying the COVID. Bastos and his colleagues[11] have also explored the accuracy of serological tests for COVID-19 and have performed a systematic review on the subject matter.

The power of deep learning and its ability to extract the correct distinct features thus substantially aiding in image classification of COVID amidst its similarity in image with pneumonia has been explored in [12-16]. The authors including Aurora et.al[16]attributed the case study for india whilst the distinct difference in all of the study is the use of different types of images such as X-ray images, CTscan images, Radiography images, nasal swabs and different deep learning convolutional architectures including VGG16, VGG19, MobileNet, AlexNet and so forth as depicted in literatures[12-20].The main argument that we have regarding the literature is that, the research papers has used pre-trained models with transfer learning based classification where the pre-training is done on imagenet[21].However, the COVID cases vary to a larger extent from that which is why we believed that apart from using pre-trained models, custom models with aid from previous architecture would perform better as theoretically hypothesized by us.So, At length, it can be said that these research works has motivated us to come forward with a study related to COVID detection where we would be proposing semi-pre trained model with some of the layers frozen and some of the layers ready for re-training on COVID dataset.

The orientation of the rest of the study is segmented into three more sections. The Section 2 shall be utilized in discussing the dataset, dataset pre-processing, model architecture and mathematical concepts of the architecture. The section 3 shall be used to implement the architecture with experimental evaluation of result analysis as well as subsequent comparison with some of the other research papers and models.Finally, the conclusion section shall be used to put forward the next future work and concluding remarks of this thesis. 


\section{Materials and Methods}

\subsection{Dataset}

The dataset has been collected from [7] and an analysis of the dataset can be found in $[7,20]$ and later modified to form the dataset. The total dataset consists of amalgamation of training data, validation data and testing data. The training dataset consists of 546 images, the testing dataset consists of 140 images and the validation dataset for k-fold cross validation has 60 images across the dataset. The whole dataset is sub-divided to two labels of classes in the folder namely , "COVID_NO" which means the images pertaining to the folder has no COVID cases and "COVID_YES" which depicts that the images in the folder are true covid images. Figure 1 and Figure 2 shows some of the examples of COVID image data.
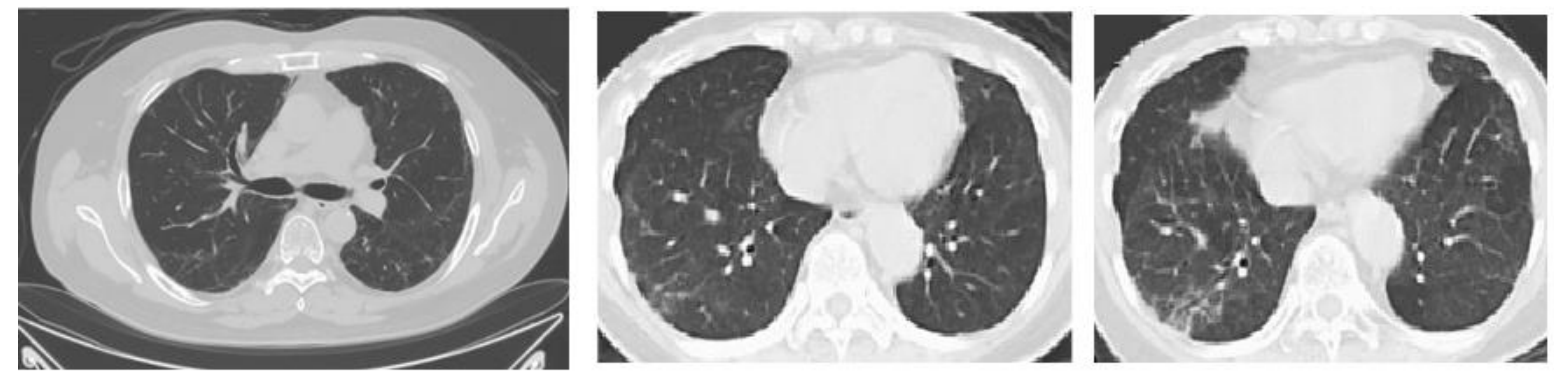

Figure 1: Sample images of CT-scan for COVID negative patients.
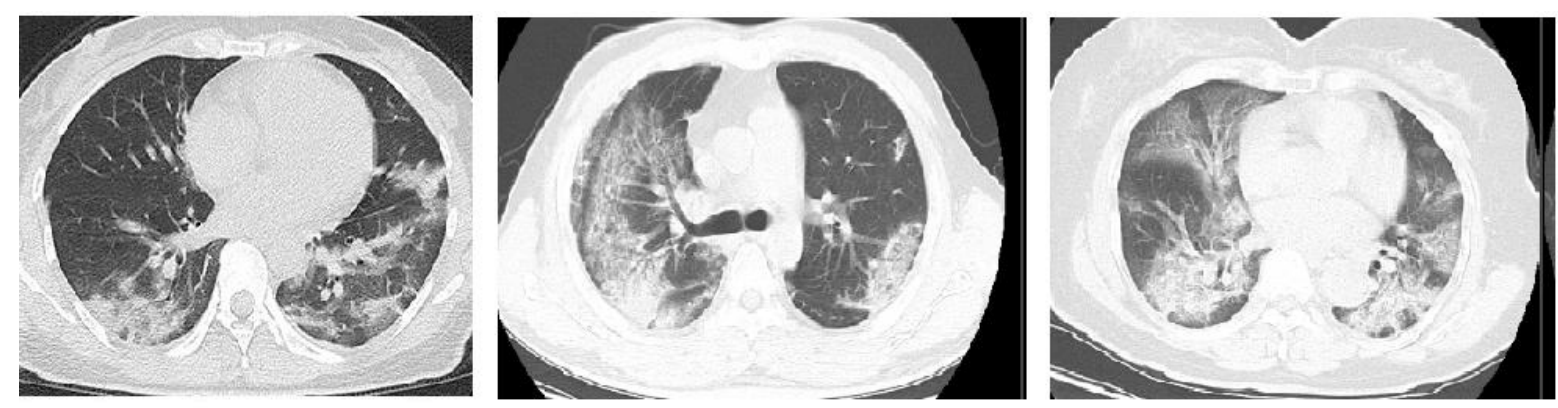

Figure 2: Sample images of CT-scan for COVID positive patients.

It is evident from the image samples that, in case of covid positive there are substantial fumes in the lungs. However, the fumes whether it is pneumonia or covid would be a substantial challenge to address. 


\subsection{Dataset Preparation}

The COVID dataset that we have downloaded varies in size and shape and some unnecessary information that we shall pre-process before feeding to a convolutional layer of networks. In the preparation stage of our dataset, we have cropped and resized our images to a smaller pixel value for obtaining higher accuracy with lower computation cost. Before finalizing our dataset we perform a data augmentation process including 40 degree rotation, grayscale channeling, normalization of the images with a shearing of the images with horizontal flip.

\subsection{Model Architecture}

Our proposed model COVIDetect is a multilayer convolutional layer given in Figure 3 that begins with input size of $224 * 224 \mathrm{rgb}$ type images connected with a convolutional layer followed by a connection with a max pooling layer. In the subsequent layers we can observe that apart from conventional convolutional layers, a batch normalization layer has been added to avoid overfitting along with a dropout layer to decrease the complexity at training time as well as overfitting problem. Except for the output consonant, all of the activation functions used were relu functions. For the output, the sigmoid function has been used.

The Rectified Linear Unit Activation ( ReLU)[23] outputs 0 for any value of $x<0$ and it has a simple linear function with slope of 1 . So, the equation for ReLUcan be depicted in equation 1 as

Equation 1

$$
\operatorname{ReLU}(x)=x, \text { for any } x \geq 0
$$

$$
\operatorname{ReLU}(x)=0, \text { for any } x<0
$$

The sigmoid function[24] outputs a curve on the basis of a designated formula with its variability with $\mathrm{x}$. So, if Sigmoid(x) is the function and e is the euler number then, the sigmoid function is equated in equation 2.

Equation 2

$$
\operatorname{Sigmoid}(x)=\frac{1}{1+e^{-x}}
$$




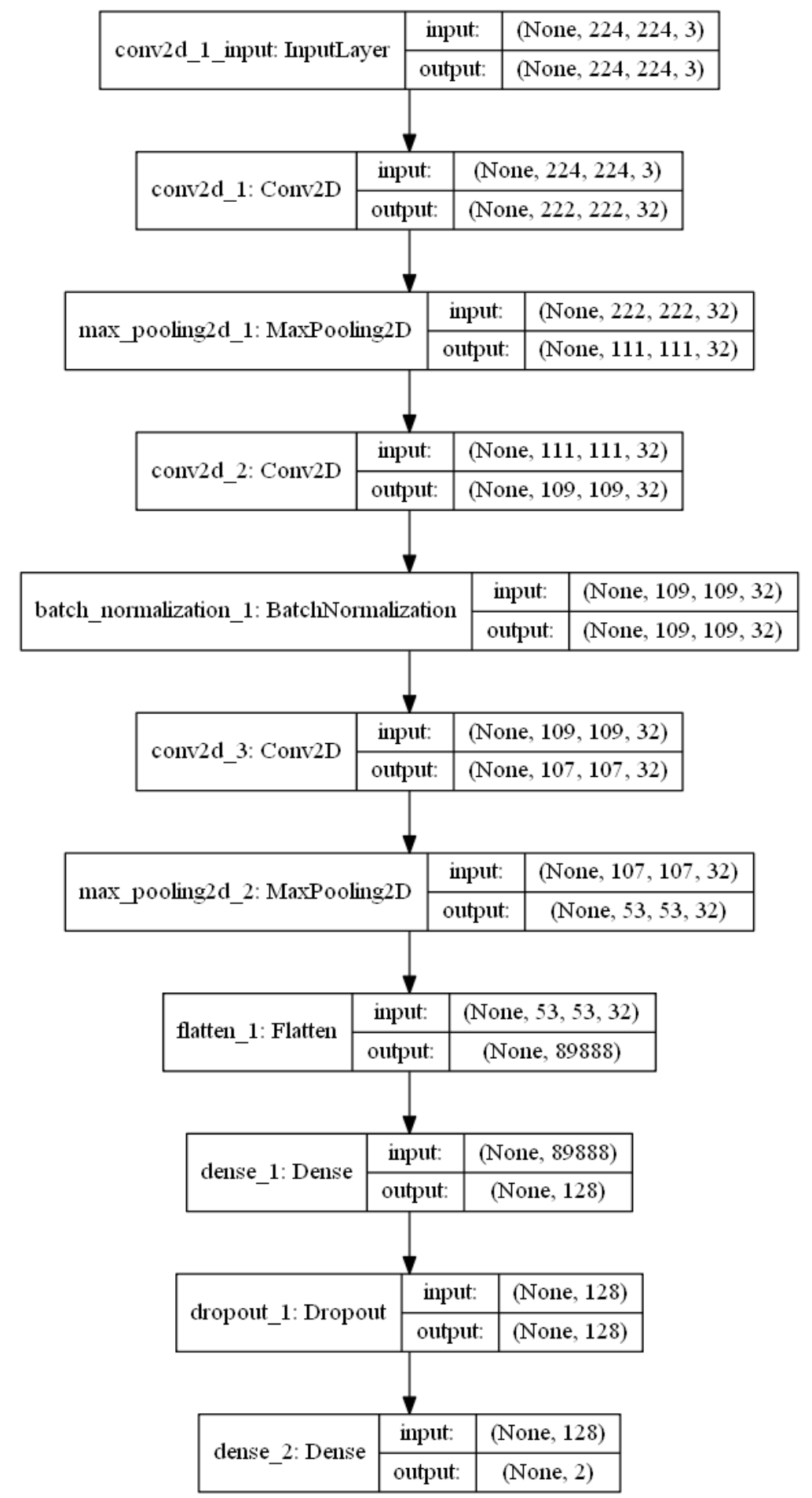

Figure 3: Our proposed Model architecture "COVIDetect" 


\subsection{Hyperparameter Tuning}

Hyperparameter tuning and choosing the optimizer plays a very important role in deep learning tasks Optimizers are basically formulas that help us to minimize the error rate with an identification function. We have used RMSprop optimizer [22] which has evolved from gradient descent algorithm. However, since the basic difference being, RMSprop being able to restrict the oscillation in vertical direction, the learning rate can be increasedsignificantly. The process involves a moving average of squared gradients to normalize the gradient to deal with the vanishing gradient problem. The formula and calculation for RMSprop is provided here in equation 3 that consists of 4 equations.

Equation 3

$$
\begin{aligned}
& G_{d w}=\beta \times G_{d w}+(1-\beta) \times d w^{2} \\
& G_{d b}=\beta \times G_{d w}+(1-\beta) \times d b^{2} \\
& W_{t}=W_{t}-\alpha \times \frac{d w}{\varepsilon+\sqrt{G_{d w}}} \\
& b=b-\alpha \times \frac{d b}{\varepsilon+\sqrt{G_{d b}}}
\end{aligned}
$$

For the purpose of using the loss function, we have used binary cross-entropy[25] as our loss function. The formula for binary cross entropy is depicted in equation 4.

Equation 4

$$
\operatorname{LossFunc}_{p}(q)=-1 / N \sum_{i=1}^{N} y_{i} \times \log \left(p\left(y_{i}\right)\right)+\left(1-y_{i}\right) \times \log \left(1-p\left(y_{i}\right)\right)
$$

where $\mathrm{y}$ is the true label and $\mathrm{p}(\mathrm{y})$ is the predicted label. We can see that this loss function is heavily dependent upon the logarithmic probability. Now in our case, our classification requires the storage of averaged positive and negative labeled classes for which we stretch the formula of equation 4 towards equation 5.

Equation 5

$$
\operatorname{FinLoss}_{p}(q)=-\frac{1}{N_{1}+N_{0}}\left[\sum_{i=1}^{N_{1}} \log \left(p\left(y_{i}\right)\right)+\sum_{i=1}^{N_{0}} \log \left(1-p\left(y_{i}\right)\right)\right]
$$




\section{Experimental Evaluation and Result Analysis}

\subsection{Experimental Evaluation}

In our experimental section, we have trained the model architecture on the dataset and noted the results which have given a fairly good result of $90 \%$ in training accuracy and $82 \%$ in validation accuracy as depicted in figure 4 . We can observe that the loss is also consistently falling as epoch is being increased with a slight case of overfitting which has been caused due to indecision of learning between pneumonia image and covid image.

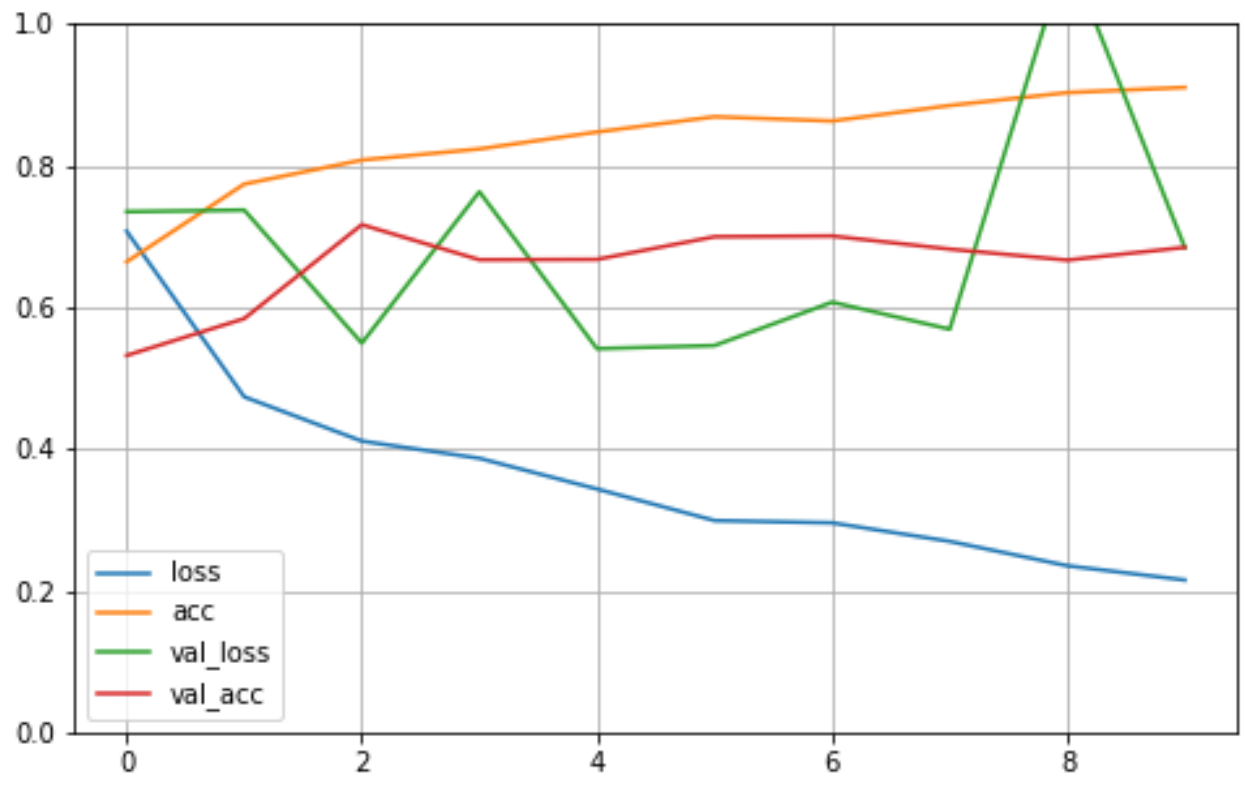

Figure 4: Training Accuracy, Training Loss, Validation Accuracy and Validation Loss of COVIDetect

The confusion matrix of COVIDetect is depicted in figure 5 that visualizes the true positive rate, false positive rates and specificity which is an important matrix towards determining the covid positive patients. From our Confusion matrix, we can compute that we have a $76.5 \%$ test accuracy , $84.3 \%$ precision, $75.6 \%$ recall and a F1 score of $81 \%$ confidence. 


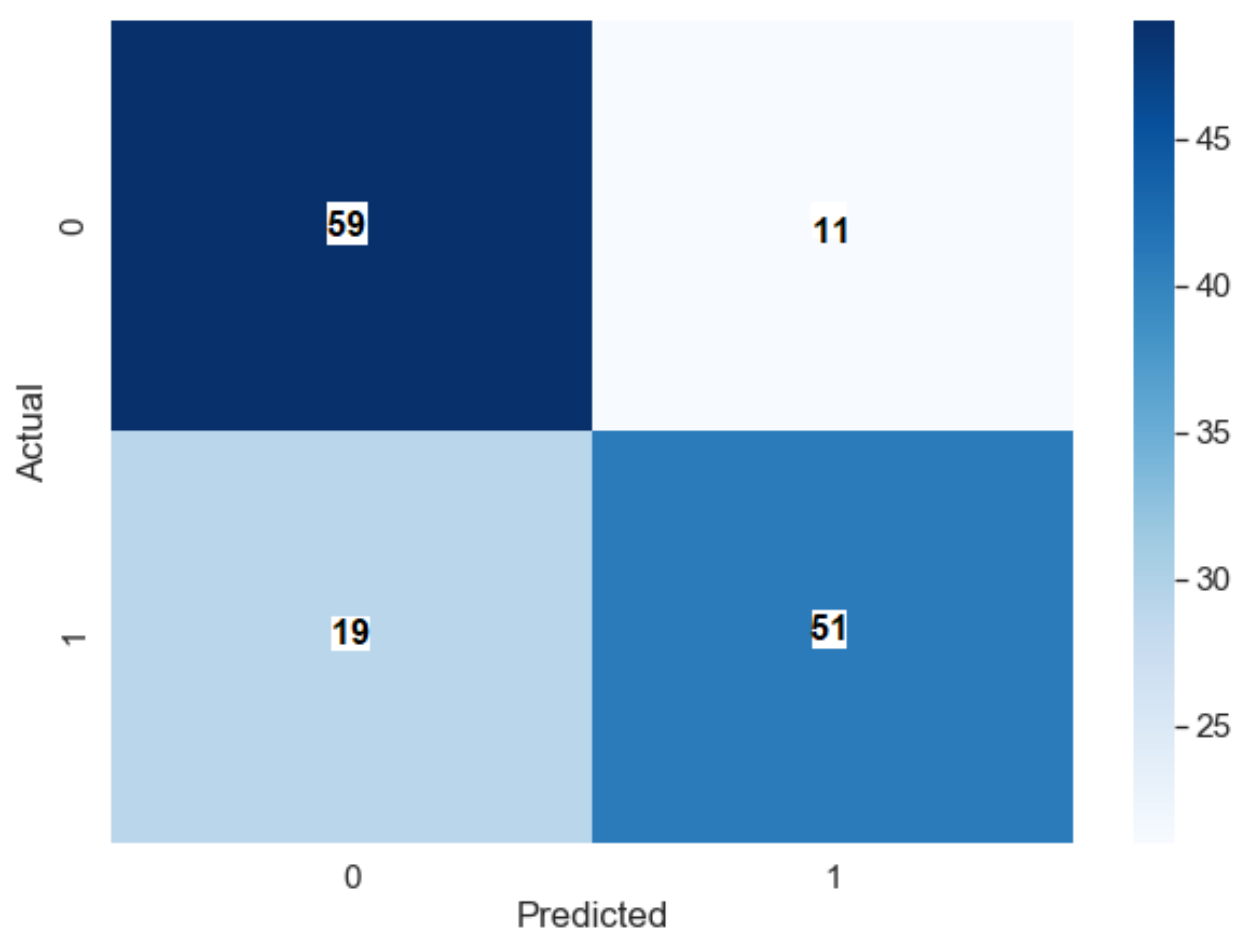

Figure 5: Confusion Matrix of COVIDetect

\subsection{Result Analysis}

Whilst COVIDetect performs the optimal classification approach, we have tweaked with 3 other versions of COVIDetect to visualize the performance and effect of batch normalization and dropout layer on image recognition. Before we perform the result analysis between other models Table 1 shall provide an insight into the variable model architecture that is being utilized for result analysis.

As per Table 1, COVIDetectV1 has no dropout and no batch normalization layer,

COVIDetectV2 has dropout layer but no batch normalization layer,

COVIDetectV3 has no dropout layer but a batch normalization layer present and finally our model COVIDetect has both dropout rate and batch normalization layer present in its model architecture as depicted in Figure 3. 
Academic Journal of Research and Scientific Publishing | Vol 2 | Issue 21

Publication Date: 5-1-2021

Table 1: A comparison of F1-score and distinguishable features of several tested model architectures.

\begin{tabular}{|l|l|l|}
\hline Model Name & Distinguishing Feature & F1-score \\
\hline COVIDetectV1 & $\begin{array}{l}\text { No Dropout layer } \\
\text { No Batch normalization layer }\end{array}$ & $66 \%$ \\
\hline COVIDetectV2 & $\begin{array}{c}\text { Dropout layer present } \\
\text { No Batch normalization layer }\end{array}$ & $68 \%$ \\
\hline COVIDetectV3 & $\begin{array}{l}\text { No Dropout layer } \\
\text { Batch normalization layer } \\
\text { present }\end{array}$ & $81 \%$ \\
\hline COVIDetect & $\begin{array}{c}\text { Dropout layer present } \\
\text { Batch normalization layer }\end{array}$ & \\
\hline
\end{tabular}

From table 1, we can see that, evidently with the introduction of dropout layer and batch normalization layer a significant increase in F1-score has been observed. Overall, although COVIDetect performs better but in some of the cases of precision and recall, COVIDetectV2 and COVIDetectV 3 also provide a better result. The Classification report can be visualized in the graphical plot of Figure 6.

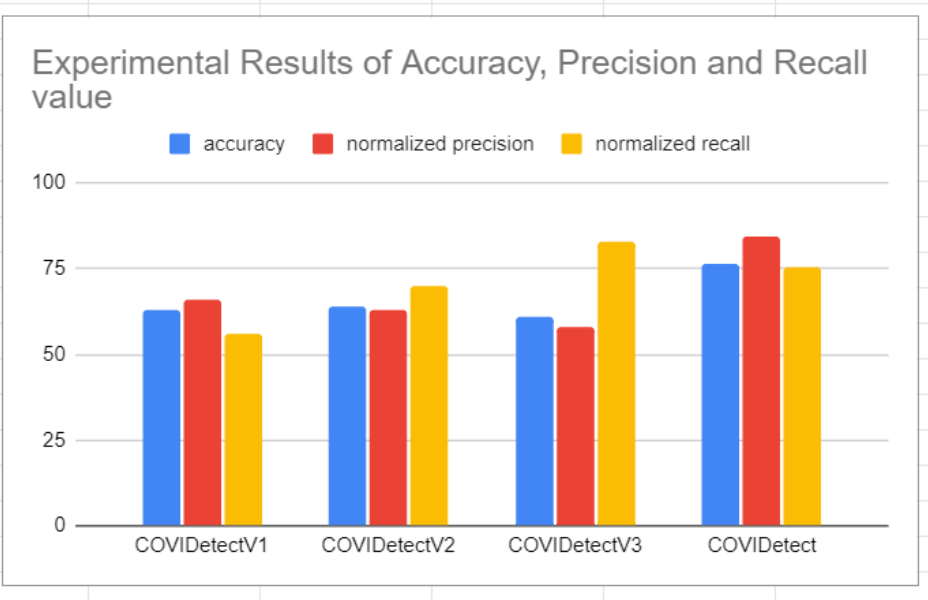

Figure 6: Experimental result comparison between architectures 


\section{Conclusion}

In COVIDetect, we stretched the need for prompt diagnosis of COVID affected patients from CT scan images. For the said task, we have exploited deep learning architectures to train and test our model on the ct-scan image dataset with a fairly good accuracy of $75.6 \%$. The model suffered from overfitting due to its acute problem of identifying the COVID affected area which in future work may be solved by exploring more complex architectures, few shot learning and federated learning. Another approach may be to increase the dataset size as, small dataset suffers from incorrect learning problems. Exploration of research can also be done in characterizing better optimizers, loss functions and tuning the hyperparameter. Finally, our contribution also performed a comparative analysis on the importance of dropout and batch normalization layer which would be insightful in carrying our future work in this domain. Our research work and dataset link is available in github for further visualization (https://github.com/militaryarman/covidetect ) .

\section{References}

[1] Gozes, O., Frid-Adar, M., Sagie, N., Zhang, H., Ji, W., \& Greenspan, H. (2020). Coronavirus detection and analysis on chest ct with deep learning. arXiv preprint arXiv:2004.02640.

[2] Horry, M. J., Chakraborty, S., Paul, M., Ulhaq, A., Pradhan, B., Saha, M., \& Shukla, N. (2020). COVID-19 detection through transfer learning using multimodal imaging data. IEEE Access, 8, 149808-149824.

[3] Narin, A., Kaya, C., \&Pamuk, Z. (2020). Automatic detection of coronavirus disease (covid-19) using X-ray images and deep convolutional neural networks. arXiv preprint arXiv:2003.10849.

[4] Bhattacharya, S., Maddikunta, P. K. R., Pham, Q. V., Gadekallu, T. R., Chowdhary, C. L., Alazab, M., \&Piran, M. J. (2020). Deep learning and medical image processing for coronavirus (COVID-19) pandemic: A survey. Sustainable cities and society, 102589. 
[5] Chen, J., Li, K., Zhang, Z., Li, K., \& Yu, P. S. (2020). A survey on applications of artificial intelligence in fighting against covid-19. arXiv preprint arXiv:2007.02202.

[6] Ulhaq, A., Born, J., Khan, A., Gomes, D. P. S., Chakraborty, S., \& Paul, M. (2020). Covid-19 control by computer vision approaches: A survey. IEEE Access, 8, 179437179456.

[7] Zhao, J., Zhang, Y., He, X., \&Xie, P. (2020). COVID-CT-Dataset: a CT scan dataset about COVID-19. arXiv preprint arXiv:2003.13865.

[8] Zobaed, S. M., Salehi, M. A., Zomaya, A., \&Sakr, S. (2019). Big Data in the Cloud.

[9] Quer, G., Radin, J. M., Gadaleta, M., Baca-Motes, K., Ariniello, L., Ramos, E., ...\&Steinhubl, S. R. (2020). Wearable sensor data and self-reported symptoms for COVID-19 detection. Nature Medicine, 1-5.

[10] Vandenberg, O., Martiny, D., Rochas, O., van Belkum, A., \&Kozlakidis, Z. (2020). Considerations for diagnostic COVID-19 tests. Nature Reviews Microbiology, 1-13.

[11] Bastos, M. L., Tavaziva, G., Abidi, S. K., Campbell, J. R., Haraoui, L. P., Johnston, J. C., ... \&Menzies, D. (2020). Diagnostic accuracy of serological tests for covid-19: systematic review and meta-analysis. $b m j, 370$.

[12] Dias, S. B., Hadjileontiadou, S. J., Diniz, J., \&Hadjileontiadis, L. J. (2020). DeepLMS: a deep learning predictive model for supporting online learning in the Covid19 era. Scientific reports, 10(1), 1-17.

[13] Ni, Q., Sun, Z. Y., Qi, L., Chen, W., Yang, Y., Wang, L., ...\& Zhou, Z. (2020). A deep learning approach to characterize 2019 coronavirus disease (COVID-19) pneumonia in chest CT images. European radiology, 30(12), 6517-6527.

[14] Xu, X., Jiang, X., Ma, C., Du, P., Li, X., Lv, S., ..\&\& Lang, G. (2020). A deep learning system to screen novel coronavirus disease 2019 pneumonia. Engineering.

[15] Ramchandani, A., Fan, C., \&Mostafavi, A. (2020). Deepcovidnet: An interpretable deep learning model for predictive surveillance of covid-19 using heterogeneous features and their interactions. IEEE Access, 8, 159915-159930. 
[16] Arora, P., Kumar, H., \&Panigrahi, B. K. (2020). Prediction and analysis of COVID19 positive cases using deep learning models: A descriptive case study of India. Chaos, Solitons\& Fractals, 139, 110017.

[17] Elsheikh, A. H., Saba, A. I., AbdElaziz, M., Lu, S., Shanmugan, S., Muthuramalingam, T., ...\&Shehabeldeen, T. A. (2020). Deep learning-based forecasting model for COVID-19 outbreak in Saudi Arabia. Process Safety and Environmental Protection, 149, 223-233.

[18] Khan, I. U., \&Aslam, N. (2020). A deep-learning-based framework for automated diagnosis of COVID-19 using X-ray images. Information, 11(9), 419.

[19] Apostolopoulos, I. D., \&Mpesiana, T. A. (2020). Covid-19: automatic detection from x-ray images utilizing transfer learning with convolutional neural networks. Physical and Engineering Sciences in Medicine, 1.

[20] He, X., Yang, X., Zhang, S., Zhao, J., Zhang, Y., Xing, E., \&Xie, P. (2020). Sample-Efficient Deep Learning for COVID-19 Diagnosis Based on CT Scans. medRxiv. [21] Krizhevsky, A., Sutskever, I., \& Hinton, G. E. (2017). Imagenet classification with deep convolutional neural networks. Communications of the ACM, 60(6), 84-90.

[22]Tieleman, T., \& Hinton, G. (2012). Lecture 6.5-rmsprop: Divide the gradient by a running average of its recent magnitude. COURSERA: Neural networks for machine learning, 4(2), 26-31.

[23] Li, Y., Yuan, Y. (2017). Convergence analysis of two-layer neural networks with relu activation. Advances in neural information processing systems, 30, 597-607.

[24] Marreiros, A. C., Daunizeau, J., Kiebel, S. J., \&Friston, K. J. (2008). Population dynamics: variance and the sigmoid activation function. Neuroimage, 42(1), 147-157.

[25] Ramos, D., Franco-Pedroso, J., Lozano-Diez, A., \& Gonzalez-Rodriguez, J. (2018). Deconstructing cross-entropy for probabilistic binary classifiers. Entropy, 20(3), 208.

Copyright (C) 2021 Md. Mobarak Hossain, Muhammad Usama Islam, Dr. Mohammod AbulKashem, AJRSP. This is an open-access article distributed under the terms of the Creative Commons Attribution License (CC BY NC). 